\title{
Choroid plexus carcinoma
}

INSERM

\section{Source}

INSERM. (1999). Orphanet: an online rare disease and orphan drug data base. Choroid plexus carcinoma. ORPHA:251899

Choroid plexus carcinoma is a rare and highly aggressive malignant type of choroid plexus tumor (see this term) occurring almost exclusively in children, presenting with cerebrospinal fluid obstruction in the lateral ventricles (most common), the fourth and third ventricles or in multiple ventricles, leading to hydrocephalus and increased intracranial pressure, and manifesting with nausea, vomiting, abnormal eye movements, gait impairment, seizures and enlarg ed head circumference. 